\title{
Development and utility assessment of a machine learning bloodstream infection classifier in pediatric patients receiving cancer treatments
}

\author{
Lillian Sung ${ }^{1 *}$ D, Conor Corbin ${ }^{2}$, Ethan Steinberg ${ }^{2}$, Emily Vettese $^{1}$, Aaron Campigotto ${ }^{3}$, Loreto Lecce ${ }^{4}$,
} George A. Tomlinson ${ }^{5}$ and Nigam Shah ${ }^{2}$

\begin{abstract}
Background: Objectives were to build a machine learning algorithm to identify bloodstream infection (BSI) among pediatric patients with cancer and hematopoietic stem cell transplantation (HSCT) recipients, and to compare this approach with presence of neutropenia to identify BSI.

Methods: We included patients 0-18 years of age at cancer diagnosis or HSCT between January 2009 and November 2018. Eligible blood cultures were those with no previous blood culture (regardless of result) within 7 days. The primary outcome was BSI. Four machine learning algorithms were used: elastic net, support vector machine and two implementations of gradient boosting machine (GBM and XGBoost). Model training and evaluation were performed using temporally disjoint training (60\%), validation (20\%) and test (20\%) sets. The best model was compared to neutropenia alone in the test set.

Results: Of 11,183 eligible blood cultures, 624 (5.6\%) were positive. The best model in the validation set was GBM, which achieved an area-under-the-receiver-operator-curve (AUROC) of 0.74 in the test set. Among the 2236 in the test set, the number of false positives and specificity of GBM vs. neutropenia were 508 vs. 592 and 0.76 vs. 0.72 respectively. Among 139 test set BSIs, six (4.3\%) non-neutropenic patients were identified by GBM. All received antibiotics prior to culture result availability.

Conclusions: We developed a machine learning algorithm to classify BSI. GBM achieved an AUROC of 0.74 and identified $4.3 \%$ additional true cases in the test set. The machine learning algorithm did not perform substantially better than using presence of neutropenia alone to predict BSI.
\end{abstract}

Keywords: Machine learning, Classifier, Bloodstream infection, Children, Cancer

\footnotetext{
* Correspondence: lillian.sung@sickkids.ca

'Division of Haematology/Oncology, The Hospital for Sick Children, 555

University Avenue, Toronto, Ontario M5G1X8, Canada

Full list of author information is available at the end of the article
}

(c) The Author(s). 2020 Open Access This article is licensed under a Creative Commons Attribution 4.0 International License, which permits use, sharing, adaptation, distribution and reproduction in any medium or format, as long as you give appropriate credit to the original author(s) and the source, provide a link to the Creative Commons licence, and indicate if changes were made. The images or other third party material in this article are included in the article's Creative Commons licence, unless indicated otherwise in a credit line to the material. If material is not included in the article's Creative Commons licence and your intended use is not permitted by statutory regulation or exceeds the permitted use, you will need to obtain permission directly from the copyright holder. To view a copy of this licence, visit http://creativecommons.org/licenses/by/4.0/ The Creative Commons Public Domain Dedication waiver (http://creativecommons.org/publicdomain/zero/1.0/) applies to the data made available in this article, unless otherwise stated in a credit line to the data. 


\section{Background}

Over the last few decades, continued improvement in survival for children with cancer has been observed [1]. Favorable survival outcomes have arisen from better risk stratification, improved understanding of the biology of pediatric cancer and intensification of therapy for some cancer types. Supportive care is also an integral component of cancer management. One of the most important toxicities of cancer treatment is bloodstream infection (BSI), defined as a microbial pathogen isolated from a blood culture. BSIs are important because they are responsible for considerable morbidity, healthcare utilization and treatment-related mortality [2, 3]. More specifically, BSIs may result in infection-related mortality in children who might otherwise be cured $[4,5]$. Patients without cancer undergoing hematopoietic stem cell transplantation (HSCT) are also at risk for life-threatening BSI [6]. Identifying the risk of BSI is important as those at lower risk may benefit from less intensive interventions such as outpatient management of fever, while those at higher risk may benefit from more intensive interventions such as broader empiric antibiotics or antibacterial prophylaxis [7, 8].

Even among children receiving identical chemotherapy, the risk of BSI is highly variable [2, 9]. Fever occurring during severe neutropenia (typically defined as an absolute neutrophil count (ANC) less than $0.5 \times 10^{9} / \mathrm{L}$ ) was one of the earliest identified risk factors that predicted lifethreatening BSI in patients receiving cancer treatments [10]. While neutropenia is an important risk factor, other factors including other laboratory parameters, bone marrow disease, underlying cancer type, treatments, inpatient status and comorbidities are also thought to be important [11]. While multiple risk stratification schemas have been developed, primarily in the setting of fever and neutropenia (FN), none are universally applicable. More specifically, rules developed in one context may not be valid in a different context [11]. Further, most rules are applied to patients with FN and thus, will miss episodes of BSI occurring in non-neutropenic patients.

The advent of at least two developments may offer both improved and individualized risk prediction for BSI in pediatric patients receiving cancer treatments. First, the transition to electronic health records (EHR) in many pediatric cancer centers in high income countries allows an opportunity to capitalize on these data at little incremental cost [12-14]. Second, machine learning approaches have gained popularity with the introduction of more powerful computing ability combined with development of newer learning algorithms [15]. Together, these developments may permit the creation of new classifiers or machine learning algorithms to detect BSI. If successful, such a classifier could be useful in multiple ways. First, it could supplant using neutropenia as the primary indication to start empiric antibiotics in patients with fever. Second, it could be used in conjunction with neutropenia to identify additional patients who may benefit from empiric antibiotics.

The objectives were to build a machine learning algorithm to identify BSI among children and adolescents with cancer and pediatric HSCT recipients, and to compare this approach with presence of neutropenia to identify BSI.

\section{Methods}

We conducted a retrospective study using data in the EHR (Allscripts) and a separate pediatric cancer database at The Hospital for Sick Children (SickKids), Toronto, Canada. The study was approved by the Research Ethics Board at SickKids (SickKids REB); the need for informed consent was waived given the retrospective nature of the study.

\section{Eligibility}

Eligible patients were 0 to 18 years of age at cancer diagnosis or HSCT (for those without cancer) in whom the diagnosis (or HSCT) occurred between January 1, 2009 and November 6, 2018.

Eligible blood cultures were those in patients without a previous blood culture (regardless of result) within 7 days prior to attempt to capture "new" potentially infectious episodes. This approach also addressed the scenario where multiple cultures were taken and only one was positive, and excluded negative cultures taken after initiation of empiric antibiotics for FN (presuming a culture was obtained prior to initiating antibiotics). We excluded blood cultures obtained prior to 28 days before either cancer diagnosis or HSCT (in those without cancer).

\section{Procedures}

Cancer-specific variables including cancer diagnosis details, relapse date and allogeneic or autologous HSCT dates were obtained from a divisional pediatric cancer database maintained by trained dedicated data managers. Down syndrome status was identified through chart review. Microbiology results and all other features were obtained from the EHR.

\section{Outcomes}

The primary outcome (label) was BSI. BSI was defined as a positive blood culture that was not a contaminant with a common commensal. Common commensals were those delineated by the National Healthcare Safety Network - Centers for Disease Control list of common commensals [16]. The common commensal list was modified to exclude viridans group streptococci given their known association with sepsis syndrome in children and adolescents with cancer [17]. Multiple positive cultures for common commensals were classified as BSI (rather than 
a contaminant) if two or more positive cultures occurred on the same day or 1 day apart [16].

\section{Potential predictors (features)}

Feature engineering was conducted based upon variables expected to be potentially associated with BSI based on previous research $[2,9,18-21]$ and clinical impression. Demographic variables included sex, age, Down syndrome, cancer diagnosis (categorized using the International Classification of Childhood Cancer main category [22]), ordinal cancer diagnosis (for example, primary or secondary cancer), relapse status and previous allogeneic or autologous HSCT at the time the culture was obtained.

The culture location (clinic, emergency department, intensive care unit or hospital ward) was identified. Hospital encounters, pathology tests and radiology tests within 28 days were counted. Hospital encounter features were the number of emergency department visits, number of admissions and total number of encounters including clinic visits. In terms of blood bank utilization, the number of platelet and red blood cell transfusions within the previous 7 days were included. Another feature was the number of prior positive blood cultures in the previous 365 days including common commensals. Antimicrobial exposure within the previous 7 days was also considered. More specifically, administration of systemic antibacterial agents used for the empiric management of FN and systemic antifungal agents were included. Administration one and 2 days prior to the blood culture and the number of days received over the previous 7 days were calculated. These same metrics were calculated for levofloxacin and caspofungin because of their utilization as infection prophylaxis in pediatric patients with cancer [23, 24].

In terms of laboratory values, we evaluated results in the $10,080 \mathrm{~min}$ prior to the culture (seven days) and specifically focused on the following: hematology: white blood cell count, ANC (neutrophils plus bands) and neutropenia (ANC less than $0.5 \times 10^{9} / \mathrm{L}$ ); and chemistry: blood urea nitrogen, creatinine, renal failure (creatinine at least 1.5 times upper limit of normal), albumen, alanine aminotransferase, glucose, lactate, $\mathrm{pH}$ (arterial, capillary and venous) and sodium. For actual values, four quantities were evaluated: 0 to $<24 \mathrm{~h}$ prior, 24 to $<48 \mathrm{~h}$ prior, average over the 7 days and either the minimum or the maximum over the 7 days. If the ANC within 24 $\mathrm{h}$ of the blood culture was missing, we imputed the ANC from one, two, or 3 days prior in that order. For most laboratory values, the minimum or maximum was chosen based upon the extreme associated with sicker patients. However, since both high and low glucose and sodium can be deleterious, both the maximum and minimum were calculated in these cases. If the same test was performed multiple times within a 24 -h period, the value closest to the culture (the later result) was used and thus, only one value per day contributed to 0 to < $24 \mathrm{~h}$ prior, 24 to $<48 \mathrm{~h}$ prior and average values.

\section{Analysis}

Baseline characteristics were compared between the BSI and non-BSI groups using the Student's t-test for continuous variables and the chi square test for categorical variables. The data set was divided into training (60\%), validation $(20 \%)$ and test $(20 \%)$ sets separated sequentially in time to avoid look-ahead bias.

Four machine learning algorithms were used, namely elastic net, support vector machine and two implementations of gradient boosting machine (GBM and XGBoost). These were implemented using the Caret package in $R$ [25]. As a general strategy, the models were trained and the parameters were tuned using the training set. The models were then implemented in the validation set and a single model was chosen to be applied to the test set. Model selection was based upon the area-under-the-receiver-operator-curve (AUROC) and diagnostic test properties (sensitivity, specific, positive predictive value, and negative predictive value). In order to compare models, we a priori decided that a new algorithm would be unacceptable if it resulted in more false negatives (failed to detect BSI) than using neutropenia alone. Thus, we set the diagnostic testing threshold such that the number of false negatives would match the number of false negatives using neutropenia within the previous $24 \mathrm{~h}$. Once a model was selected, the model was re-trained and parameters were re-tuned using the combined training and validation sets $(80 \%)$. The final model was then applied to the test set.

For data preparation, laboratory features that were missing in more than $80 \%$ of eligible blood cultures were removed. Other missing values were imputed singly using chained equations via the MICE package in R [26]. Imputation was performed separately in the training, validation and test sets. Other model pre-processing steps consisted of centering, scaling and removing near-zero variance features. A grid search was used for parameter tuning. Models were trained using five cross-validation folds repeated five times in which the metric monitored was the AUROC. Both in-sample (cross-validation) and out-of-sample AUROCs were reported. All analyses were performed using $\mathrm{R}$ studio version 3.6.1, The $\mathrm{R}$ Foundation for Statistical Computing.

\section{Results}

There were 11,183 eligible blood cultures from 2306 patients included in the analysis. Overall, the number of positive BSI was $624(5.6 \%)$. Baseline characteristics and features are shown in Tables 1, 2, 3 stratified by BSI. 
Table 1 Demographics of the Cohort Stratified by Bloodstream Infection ( $N=11,183$ cultures)

\begin{tabular}{|c|c|c|c|}
\hline & Negative & Positive & $P^{*}$ \\
\hline$n$ & 10,559 & 624 & \\
\hline Male Sex & $5841(55.3)$ & $330(52.9)$ & 0.252 \\
\hline Mean Age (SD) & $7.10(4.82)$ & $7.47(5.11)$ & 0.062 \\
\hline Down Syndrome & $263(2.5)$ & $26(4.2)$ & 0.015 \\
\hline ICCC Category & & & $<0.001$ \\
\hline $\begin{array}{l}\text { I. Leukemias, Myeloproliferative Diseases and } \\
\text { Myelodysplastic Diseases }\end{array}$ & $5070(48.0)$ & $403(64.6)$ & \\
\hline II. Lymphomas and Reticuloendothelial Neoplasms & $1325(12.5)$ & $56(9.0)$ & \\
\hline III. Central Nervous System Tumors & $1223(11.6)$ & $52(8.3)$ & \\
\hline IV. Neuroblastoma & $899(8.5)$ & $48(7.7)$ & \\
\hline V. Retinoblastoma & $127(1.2)$ & $3(0.5)$ & \\
\hline VI. Renal Tumors & $248(2.3)$ & $2(0.3)$ & \\
\hline VII. Hepatic Tumors & $168(1.6)$ & $4(0.6)$ & \\
\hline VIII. Malignant Bone Tumors & $528(5.0)$ & $15(2.4)$ & \\
\hline IX. Soft-tissue and Other Extraosseous Sarcomas & $573(5.4)$ & $20(3.2)$ & \\
\hline X. Germ Cell Tumors & $146(1.4)$ & $3(0.5)$ & \\
\hline XI. Other Malignant Epithelial Neoplasms & $103(1.0)$ & $6(1.0)$ & \\
\hline XII. Other and unspecified malignant neoplasms & $19(0.2)$ & $1(0.2)$ & \\
\hline Not cancer undergoing HSCT & $130(1.2)$ & $11(1.8)$ & \\
\hline Ordinal Cancer & & & 0.904 \\
\hline 1 & $10,446(98.9)$ & $617(98.9)$ & \\
\hline 2 & $105(1.0)$ & $7(1.1)$ & \\
\hline 3 or 4 & $8(0.1)$ & $0(0.0)$ & \\
\hline Relapse & $1132(10.7)$ & $134(21.5)$ & $<0.001$ \\
\hline \multicolumn{4}{|l|}{ Prior Hematopoietic Stem Cell Transplant } \\
\hline Allogeneic & $739(7.0)$ & $94(15.1)$ & $<0.001$ \\
\hline Autologous & $914(8.7)$ & $61(9.8)$ & 0.373 \\
\hline
\end{tabular}

Abbreviations: SD Standard deviation, ICCC International Classification of Childhood Cancer, HSCT Hematopoietic stem cell transplantation ${ }^{*} P$ values calculated using Student's t-test for continuous variables and chi square test for categorical variables

More detailed cancer diagnosis is shown in Additional file 1: Appendix 1; the most common cancer was lymphoid leukemia (4140, 37.0\%). Additional file 1: Appendix 2 shows the lab values that were removed because they were missing in over $80 \%$ of blood culture episodes; most related to lactate dehydrogenase and arterial, capillary or venous $\mathrm{pH}$ values. Table 4 and Additional file 1: Appendix 3 illustrate the isolates associated with BSI. The most common pathogens were coagulase negative staphylococci, viridans group streptococci, Escherichia species, Staphylococcus aureus and Pseudomonas aeruginosa in descending order. Additional file 1: Appendix 4 illustrates the number of eligible blood cultures, number of unique patients, number of positive cultures and number of unique patients with positive cultures within the training ( $n=6710$ cultures), validation $(n=$
2237 cultures $)$ and test $(n=2236$ cultures $)$ sets separately.

Additional file 1: Appendix 5 illustrates model performance in the training and validation sets. The insample cross-validation AUROC ranged from 0.71 to 0.79 across the four models with XGBoost having the highest cross-validation AUROC of 0.79. In the validation set, the out-of-sample AUROC was 0.77 for elastic net, GBM and XGBoost. Thus, model choice relied upon diagnostic testing properties. The number of false negatives in the validation set with the neutropenia model was 47 of 2237 cultures. Setting the same number of false negatives across all four models, accuracy (fraction of predictions that were correct) was highest for GBM (0.74) and lowest for support vector machine (0.48). In evaluating kappa, sensitivity, specificity, positive predictive value and negative predictive value, GBM was the same or 
Table 2 Preceding Healthcare Encounters, Tests, Blood Bank Utilization and Systemic Antibiotic Administration ( $N=11,183$ cultures)

\begin{tabular}{|c|c|c|c|}
\hline & Negative & Positive & $P^{*}$ \\
\hline N & 10,559 & 624 & \\
\hline Location where Culture Obtained & & & $<0.001$ \\
\hline Clinic & $1346(12.7)$ & $62(9.9)$ & \\
\hline Emergency Department & $4877(46.2)$ & $219(35.1)$ & \\
\hline Intensive Care Unit & $329(3.1)$ & $9(1.4)$ & \\
\hline Hospital Ward & $4007(37.9)$ & $334(53.5)$ & \\
\hline \multicolumn{4}{|l|}{ Healthcare Encounters within 28 Days } \\
\hline Mean Number Emergency Department Visits (SD) & $0.35(0.62)$ & $0.28(0.54)$ & 0.006 \\
\hline Mean Number Admissions (SD) & $0.53(0.71)$ & $0.57(0.67)$ & 0.178 \\
\hline Mean All Encounters (SD) & $3.95(3.32)$ & $3.86(3.06)$ & 0.474 \\
\hline \multicolumn{4}{|l|}{ Tests Performed within 28 Days } \\
\hline Mean Pathology Specimens (SD) & $0.24(0.77)$ & $0.39(1.04)$ & $<0.001$ \\
\hline Mean Radiology Tests (SD) & $1.12(2.07)$ & $1.37(2.14)$ & 0.003 \\
\hline \multicolumn{4}{|l|}{ Blood Bank Utilization within 7 Days } \\
\hline Mean Platelet Transfusions (SD) & $0.40(1.20)$ & $1.15(1.78)$ & $<0.001$ \\
\hline Mean Red Cell Transfusions (SD) & $0.28(0.80)$ & $0.53(0.91)$ & $<0.001$ \\
\hline \multicolumn{4}{|l|}{ Previous Positive Blood Cultures 7-365 Days } \\
\hline Mean Positive Cultures (SD) & $0.65(1.95)$ & $1.18(2.22)$ & $<0.001$ \\
\hline \multicolumn{4}{|l|}{ Systemic Antibiotics within 7 Days } \\
\hline FN Antibiotics One Day Prior (\%) & $273(2.6)$ & $11(1.8)$ & 0.255 \\
\hline FN Antibiotics Two Days Prior (\%) & $250(2.4)$ & $14(2.2)$ & 0.950 \\
\hline Mean Days FN Antibiotics (SD) & $0.24(0.86)$ & $0.35(0.94)$ & 0.001 \\
\hline Antifungal One Day Prior (\%) & $1008(9.5)$ & $210(33.7)$ & $<0.001$ \\
\hline Antifungal Two Days Prior (\%) & $936(8.9)$ & $208(33.3)$ & $<0.001$ \\
\hline Mean Days Antifungal (SD) & $0.56(1.56)$ & $1.94(2.56)$ & $<0.001$ \\
\hline Levofloxacin One Day Prior (\%) & $36(0.3)$ & $8(1.3)$ & 0.001 \\
\hline Levofloxacin Two Days Prior (\%) & $45(0.4)$ & $5(0.8)$ & 0.291 \\
\hline Mean Days Levofloxacin (SD) & $0.02(0.25)$ & $0.08(0.50)$ & $<0.001$ \\
\hline Caspofungin One Day Prior (\%) & $380(3.6)$ & $55(8.8)$ & $<0.001$ \\
\hline Caspofungin Two Days Prior (\%) & 349 (3.3) & $56(9.0)$ & $<0.001$ \\
\hline Mean Days Caspofungin (SD) & $0.22(1.01)$ & $0.56(1.58)$ & $<0.001$ \\
\hline
\end{tabular}

Abbreviations: SD Standard deviation, FN Fever and neutropenia

* $P$ values calculated using Student's t-test for continuous variables and chi square test for categorical variables

better than the other approaches and thus, the GBM model was chosen. When the model was re-trained and parameters were re-tuned using GBM in the training and validation sets combined, the in-sample AUROC from cross-validation was 0.78. Additional file 1: Appendix 6 illustrates the 20 most important features contributing to the final model. Neutropenia within $24 \mathrm{~h}$ was not included in this list.

Table 5 shows the performance of GBM in the test set and compares it to the neutropenia model using the threshold derived when setting the number of false negatives to be the same as in the neutropenia model (52 of 2236 cultures). Specificity was 0.76 with GBM compared to 0.72 with the neutropenia model, resulting in 508 false positives with GBM and 592 with the neutropenia model (difference of 84 cases). Among the 139 with BSI in the test set, 81 were positive by both GBM and neutropenia, 46 were negative by both and six were missed by each model. Among the 2097 with negative BSI, 1356 were negative by both GBM and neutropenia, 359 were positive by both, 149 were false positives with GBM and 233 were false positives with neutropenia. The AUROC of GBM in the test set was 0.74 .

If GBM was applied in addition to neutropenia as the criteria to initiate empiric antibacterial agents, six nonneutropenic patients would be identified, representing $6 /$ 
Table 3 Preceding Laboratory Values within Seven Days $\left(N=11,183\right.$ cultures) ${ }^{a}$

\begin{tabular}{|c|c|c|c|}
\hline & Negative & Positive & $P^{* * *}$ \\
\hline$n$ & 10,559 & 624 & \\
\hline \multicolumn{4}{|l|}{ WBC, ANC and Neutropenia } \\
\hline Mean WBC $0-<24$ Hours (SD) & $8.39(27.04)$ & $4.38(13.54)$ & $<0.001$ \\
\hline Mean WBC 24-<48 Hours (SD) & $5.52(12.33)$ & $1.67(6.80)$ & $<0.001$ \\
\hline Mean Average WBC Prior 7 Days (SD) & $8.24(26.29)$ & $4.70(13.31)$ & 0.001 \\
\hline Mean Minimum WBC Prior 7 Days (SD) & $6.90(26.08)$ & $3.73(13.03)$ & 0.003 \\
\hline Mean ANC 0- $<24$ Hours (SD) & $4.15(6.50)$ & $2.66(4.73)$ & $<0.001$ \\
\hline Mean ANC 24- < 48 Hours (SD) & $3.38(6.32)$ & $1.10(3.21)$ & $<0.001$ \\
\hline Mean Average ANC Prior 7 Days (SD) & $3.96(5.91)$ & $2.73(4.15)$ & $<0.001$ \\
\hline Mean Minimum ANC Prior 7 Days (SD) & $3.00(5.37)$ & $1.90(3.61)$ & $<0.001$ \\
\hline Number Neutropenic $0-<24$ Hours $(\%)^{b}$ & $2745(26.0)$ & 397 (63.6) & $<0.001$ \\
\hline Number Neutropenic $24-<48$ Hours (\%) & $1146(10.9)$ & $253(40.5)$ & $<0.001$ \\
\hline \multicolumn{4}{|l|}{ Renal Function } \\
\hline Mean BUN 0- <24 Hours (SD) & $4.41(2.79)$ & $4.88(2.85)$ & 0.001 \\
\hline Mean BUN 24- < 48 Hours (SD) & $4.65(3.34)$ & $5.22(3.04)$ & 0.011 \\
\hline Mean Average BUN Prior 7 Days (SD) & $4.47(2.60)$ & $4.86(2.41)$ & 0.001 \\
\hline Mean Maximum BUN Prior 7 Days (SD) & $5.07(3.20)$ & $5.66(3.01)$ & $<0.001$ \\
\hline Mean Creatinine $0-<24$ Hours (SD) & $37.35(35.55)$ & $34.16(14.24)$ & 0.039 \\
\hline Mean Creatinine 24- < 48 Hours (SD) & $36.32(39.38)$ & $34.25(14.47)$ & 0.367 \\
\hline Mean Average Creatinine Prior 7 Days (SD) & $36.93(35.50)$ & $34.18(13.66)$ & 0.062 \\
\hline Mean Maximum Creatinine Prior 7 Days (SD) & $39.67(40.39)$ & $37.93(16.18)$ & 0.299 \\
\hline Renal Failure $0-<24$ Hours (\%) ${ }^{b}$ & $122(1.2)$ & $2(0.3)$ & 0.082 \\
\hline Renal Failure $24-<48$ Hours $(\%)^{b}$ & $48(0.5)$ & $1(0.2)$ & 0.441 \\
\hline Renal Failure Prior 7 Days (\%) & $164(1.6)$ & $5(0.8)$ & 0.184 \\
\hline \multicolumn{4}{|l|}{ Other Laboratory Values } \\
\hline Mean Albumen 0-<24 Hours (SD) & $35.02(6.33)$ & $34.22(5.99)$ & 0.102 \\
\hline Mean Average Albumen Prior 7 Days (SD) & $35.44(6.13)$ & $34.57(5.36)$ & 0.012 \\
\hline Mean Minimum Albumen Prior 7 Days (SD) & $34.08(6.64)$ & $33.01(5.82)$ & 0.005 \\
\hline Mean ALT 0- $<24$ Hours (SD) & $81.28(159.48)$ & $78.32(79.34)$ & 0.785 \\
\hline Mean Average ALT Prior 7 Days (SD) & $77.01(123.52)$ & $78.70(89.95)$ & 0.781 \\
\hline Mean Maximum Prior 7 Days ALT (SD) & 87.07 (146.59) & $92.97(116.53)$ & 0.415 \\
\hline Mean Glucose 0-<24 Hours (SD) & $5.59(2.31)$ & $5.60(1.83)$ & 0.928 \\
\hline Mean Glucose 24- < 48 Hours (SD) & $5.51(2.04)$ & $5.64(2.32)$ & 0.310 \\
\hline Mean Average Glucose Prior 7 Days (SD) & $5.54(1.64)$ & $5.61(1.40)$ & 0.341 \\
\hline Mean Minimum Glucose Prior 7 Days (SD) & $5.09(1.45)$ & $4.93(1.07)$ & 0.013 \\
\hline Mean Maximum Glucose Prior 7 Days (SD) & $6.17(3.01)$ & $6.55(2.79)$ & 0.004 \\
\hline Mean Sodium 0- <24 Hours (SD) & $138.22(3.34)$ & $137.54(3.86)$ & $<0.001$ \\
\hline Mean Sodium 24- < 48 Hours (SD) & $139.13(3.38)$ & $138.97(3.27)$ & 0.434 \\
\hline Mean Average Sodium Prior 7 Days (SD) & $138.62(2.96)$ & $138.27(3.28)$ & 0.006 \\
\hline Mean Minimum Sodium Prior 7 Days (SD) & $137.59(3.20)$ & $136.65(3.68)$ & $<0.001$ \\
\hline Mean Maximum Sodium Prior 7 Days (SD) & $139.65(3.46)$ & $139.82(3.77)$ & 0.255 \\
\hline
\end{tabular}

Abbreviations: SD Standard deviation, ANC Absolute neutrophil count, WBC White blood cell count, BUN Blood urea nitrogen, ALT Alanine aminotransferase ${ }^{a}$ Units: WBC $10^{9} / \mathrm{L}, \mathrm{ANC} 10^{9} / \mathrm{L}, \mathrm{BUN} \mathrm{mmol} / \mathrm{L}$, creatinine umol/L, albumen $\mathrm{g} / \mathrm{L}$, ALT U/L, glucose $\mathrm{mmol} / \mathrm{L}$, sodium $\mathrm{mmol} / \mathrm{L}$

${ }^{\mathrm{b}}$ Neutropenia defined as ANC $<0.5 \times 10^{9} / \mathrm{L}$; renal failure defined as serum creatinine $\geq 1.5$ times upper limit of normal

*** $P$ values calculated using Student's t-test for continuous variables and chi square test for categorical variables 
Table 4 Most Common Bloodstream Infection Microorganisms

\begin{tabular}{ll}
\hline Species & Frequency \\
\hline Coagulase Negative Staphylococci & 148 \\
Viridans Group Streptococci & 140 \\
Escherichia species & 76 \\
Staphylococcus aureus & 65 \\
Pseudomonas aeruginosa & 44 \\
Enterobacter species & 40 \\
Klebsiella species & 40 \\
Micrococcus species & 23 \\
Streptococcus pneumoniae & 29 \\
Bacillus species & 21 \\
\hline
\end{tabular}

139 (4.3\%) of those with BSI. The microorganisms were as follows: coagulase negative staphylococci $(n=1)$, Acinetobacter spp. $(n=1)$, Enterobacter spp. $(n=1)$, Proteus spp. $(n=1)$, Moraxella spp. $(n=1)$ and non-albicans Candida. Five patients had high-risk FN features (had they been neutropenic) as follows: post allogeneic HSCT $(n=3)$, post autologous HSCT $(n=1)$ and induction chemotherapy for acute lymphoblastic leukemia $(n=1)$. All of these patients with high-risk features received parenteral antibiotics prior to the culture results being known while the sixth patient received oral antibiotics prior to culture result availability. Two experienced bacteria-related sepsis although none died during the episode.

\section{Discussion}

We were successful in using an institutional EHR to develop a machine learning algorithm to predict BSI. While the in-sample and the out-of-sample AUROC were reasonable, the final model did not perform substantially better than the presence of neutropenia at fever onset. Even though the model was able to identify additional BSI in non-neutropenic patients, these predictions would not have provided additional value because all of these patients had received empiric antibiotics. Provision of empiric antibiotics in non-neutropenic patients is standard in patients who appear ill or when clinicians apply their own judgement and experience in identifying higher risk patients.

The developed BSI classifier performance does not support its use as a replacement for neutropenia as an indication for empiric therapy. If used as an adjunct to neutropenia, whether the identification of six additional non-neutropenic BSI cases at the cost of 149 more false positive results is worthwhile will depend on at least two major considerations. First is the advantage of identifying non-neutropenic patients with BSI and whether deploying the model would improve clinically meaningful outcomes [27]. To this point, one of the BSI was a yeast and thus, empiric antibacterial agents would not have led to better results. On the other hand, two of these patients developed bacterial sepsis, emphasizing the importance of early antibiotics in this small cohort. In our setting, all non-neutropenic BSI patients received empiric antibiotics prior to culture result availability and thus, the algorithm would not be useful at our institution. However, such an algorithm could be useful in settings in which limited expertise and resources, or high volumes impede clinicians from deciding which non-neutropenic patients should receive empiric antibacterial agents.

The second consideration is the cost and effort to implement a machine learning algorithm into the clinical workflow of busy clinicians and commonly overburdened information technology hospital staff. Indeed, for a machine learning model to be successful, it would need to be integrated at any location in which pediatric patients receiving cancer treatments are assessed for fever including clinics, the emergency department and the inpatient ward. This complexity increases the hidden deployment cost [28]. Unless the benefit of a machine learning algorithm is clearly evident, such implementation is likely to be challenging.

There are several potential reasons why the developed algorithm did not perform substantially better than neutropenia alone. First, it is possible that the sample size was too small, particularly when considering the number of unique patients in this data set. Second, it is possible that features most important to predicting BSI were not present in this data set. A notable absence is the lack of flow chart data, which only was implemented into the EHR during the latter part of the study period and thus, could not be used in model building. Future work should focus on identifying other data that could inform a BSI classifier. In comparing classifiers and the best classifier against the neutropenia model, we chose to fix the sensitivity to ensure a new model could not miss

Table 5 Performance in Test Set $(N=2236)^{\text {a }}$

\begin{tabular}{|c|c|c|c|c|c|c|c|c|c|}
\hline & $\begin{array}{l}\text { Proportion } \\
\text { Positive }\end{array}$ & Sensitivity & Specificity & $\begin{array}{l}\text { Positive } \\
\text { Predictive } \\
\text { Value }\end{array}$ & $\begin{array}{l}\text { Negative } \\
\text { Predictive } \\
\text { Value }\end{array}$ & $\begin{array}{l}\text { True } \\
\text { Positive }\end{array}$ & $\begin{array}{l}\text { False } \\
\text { Positive }\end{array}$ & $\begin{array}{l}\text { True } \\
\text { Negative }\end{array}$ & $\begin{array}{l}\text { False } \\
\text { Negative }\end{array}$ \\
\hline $\mathrm{GBM}^{\mathrm{b}}$ & 595 (26.6\%) & 0.63 & 0.76 & 0.15 & 0.97 & 87 & 508 & 1589 & 52 \\
\hline Neutropenia & 679 (30.4\%) & 0.63 & 0.72 & 0.13 & 0.97 & 87 & 592 & 1505 & 52 \\
\hline
\end{tabular}

Abbreviation: GBM one implementation of gradient boosting machine

${ }^{a}$ Observed bloodstream infection rate 139/2236 (6.2\%) in the test set

${ }^{b}$ Test threshold chosen (probability $\left.>0.0489\right)$ to keep the number of false negatives the same as in the absolute neutrophil count $<0.5 \times 10^{9} / L$ model $(52 / 2236)$ 
more cases of bacteremia. Alternatively, we could have chosen to fix the specificity to see whether the model might have enhanced sensitivity. However, this analysis could also show worse sensitivity, a scenario that would not be considered clinically acceptable.

A strength of this study was the use of a well-curated cancer diagnosis and treatment data set combined with EHR data to build a BSI classifier. A second strength is that it complements the large literature of risk prediction models in FN by expanding the target to all pediatric cancer patients at risk for BSI. However, this study has several limitations. The analysis did not account for the correlated structure of the data in that the same patient could contribute multiple episodes although this may be less problematic since our focus was on prediction rather than inference. Second, different blood draws from the same patient could have been in the training, validation and test sets and this approach may have resulted in overly optimistic results. However, this aspect is likely less problematic as it mimics how the algorithm would be deployed in clinical practice. Finally, our data set was relatively small, and it is possible that the algorithms would have performed better had more data been available.

\section{Conclusion}

In conclusion, we developed a machine learning algorithm to classify BSI. GBM achieved an AUROC of 0.74 and identified $4.3 \%$ additional true cases in the test set. The machine learning algorithm did not perform substantially better than using the presence of neutropenia alone to predict BSI.

\section{Supplementary Information}

The online version contains supplementary material available at https://doi. org/10.1186/s12885-020-07618-2

\section{Additional file 1.}

\section{Abbreviations \\ ANC: Absolute neutrophil count; AUROC: Area-under-the-receiver-operator- curve; BSI: Bloodstream infection; EHR: Electronic health records; FN: Fever and neutropenia; HSCT: Hematopoietic stem cell transplantation; SickKids REB: Research Ethics Board at SickKids; SickKids: Hospital for Sick Children}

\section{Acknowledgements}

We would like to thank Mark Watmough, Joyce Magbitang, Rodica Balaci and Avi Kant who facilitated data acquisition. LS is supported by the Canada Research Chair in Pediatric Oncology Supportive Care.

\section{Authors' contributions}

LS developed manuscript concept, designed content and structure and wrote first draft. All authors (LS, CC, ES, EV, AC, LL, GT, NS) were involved in the conduct of study and analysis, manuscript revision for critical content and final version approval. The author(s) read and approved the final manuscript.

\section{Funding}

There was no funding for this study.
Availability of data and materials

As the data include personal health information, the data are not available publicly.

\section{Ethics approval and consent to participate}

The institutional research ethics board at the Hospital for Sick Children reviewed and approved the study (Research Ethics Board No. 1000062140). Waiver of consent has been approved.

\section{Consent for publication}

Not applicable.

\section{Competing interests}

The authors declare that they have no competing interests.

\section{Author details}

'Division of Haematology/Oncology, The Hospital for Sick Children, 555 University Avenue, Toronto, Ontario M5G1X8, Canada. ${ }^{2}$ Biomedical Informatics Research, Stanford University, Palo Alto, USA. ${ }^{3}$ Division of Infectious Diseases, The Hospital for Sick Children, Toronto, Canada. ${ }^{4}$ Division of Neonatology, The Hospital for Sick Children, Toronto, Canada.

${ }^{5}$ Department of Medicine, University Health Network, Toronto, Canada.

Received: 11 March 2020 Accepted: 6 November 2020

Published online: 13 November 2020

\section{References}

1. Canadian Cancer Society's Steering Committee on Cancer Statistics. Canadian Cancer statistics. Toronto: Canadian Cancer Society; 2011.

2. Sung L, Lange BJ, Gerbing RB, Alonzo TA, Feusner J. Microbiologically documented infections and infection-related mortality in children with acute myeloid leukemia. Blood. 2007;110(10):3532-9.

3. Lehrnbecher T, Sung L. Anti-infective prophylaxis in pediatric patients with acute myeloid leukemia. Expert Rev Hematol. 2014;7(6):819-30.

4. Gibson P, Pole JD, Lazor T, Johnston D, Portwine C, Silva M, et al. Treatment-related mortality in newly diagnosed pediatric cancer: a population-based analysis. Cancer Med. 2018;7(3):707-15.

5. Alexander S, Pole JD, Gibson P, Lee M, Hesser T, Chi SN, et al. Classification of treatment-related mortality in children with cancer: a systematic assessment. Lancet Oncol. 2015;16(16):e604-10.

6. Akinboyo IC, Young RR, Spees LP, Heston SM, Smith MJ, Chang YC, et al. Microbiology and Risk Factors for Hospital-Associated Bloodstream Infections Among Pediatric Hematopoietic Stem Cell Transplant Recipients. Open Forum Infect Dis. 2020;7(4):ofaa093.

7. Lehrnbecher T, Fisher BT, Phillips B, Alexander S, Ammann RA, Beauchemin M, Carlesse F, Castagnola E, Davis BL, Dupuis LL, Egan G, Groll AH, Haeusler GM, Santolaya M, Steinbach WJ, van de Wetering M, Wolf J, Cabral S, Robinson PD, Sung L. Guideline for Antibacterial Prophylaxis Administration in Pediatric Cancer and Hematopoietic Stem Cell Transplantation. Clin Infect Dis. 2020;71(1):226-36. https://doi.org/10.1093/cid/ciz1082.

8. Egan G, Robinson PD, Martinez JPD, Alexander S, Ammann RA, Dupuis LL, et al. Efficacy of antibiotic prophylaxis in patients with cancer and hematopoietic stem cell transplantation recipients: a systematic review of randomized trials. Cancer Med. 2019;8(10):4536-46.

9. Dix D, Cellot S, Price V, Gillmeister B, Ethier MC, Johnston DL, et al. Association between corticosteroids and infection, sepsis, and infectious death in pediatric acute myeloid leukemia (AML): results from the Canadian infections in AML research group. Clin Infect Dis. 2012;55(12):1608-14.

10. Pizzo PA. Management of Patients with Fever and Neutropenia through the arc of time: a narrative review. Ann Intern Med. 2019;170(6):389-97.

11. Phillips RS, Lehrnbecher T, Alexander S, Sung L. Updated systematic review and meta-analysis of the performance of risk prediction rules in children and young people with febrile neutropenia. PLoS One. 2012;7(5):e38300.

12. Wang W, Krishnan E. Big data and clinicians: a review on the state of the science. JMIR Med Inform. 2014;2(1):e1.

13. Hansen MM, Miron-Shatz T, Lau AY, Paton C. Big data in science and healthcare: a review of recent literature and perspectives. Contribution of the IMIA social media working group. Yearb Med Inform. 2014;9:21-6.

14. El Aboudi N, Benhlima L. Big data Management for Healthcare Systems: architecture, requirements, and implementation. Adv Bioinforma. 2018:2018: 4059018. 
15. Rajkomar A, Dean J, Kohane I. Machine learning in medicine. N Engl J Med. 2019;380(14):1347-58.

16. Central Line-Associated Bloodstream Infection (CLABSI) and non-central line-associated Bloodstream Infection Atlanta, GA: Centers for Disease Control and Prevention; 2017 [updated August 3, 2017. Available from: https://www.cdc.gov/nhsn/acute-care-hospital/clabsi/index.html.

17. Facklam R. What happened to the streptococci: overview of taxonomic and nomenclature changes. Clin Microbiol Rev. 2002;15(4):613-30.

18. Dandoy CE, Ardura MI, Papanicolaou GA, Auletta JJ. Bacterial bloodstream infections in the allogeneic hematopoietic cell transplant patient: new considerations for a persistent nemesis. Bone Marrow Transplant. 2017:52(8): 1091-106.

19. Thurman CB, Abbott M, Liu J, Larson E. Risk for health care-associated bloodstream infections in pediatric oncology patients with various malignancies. J Pediatr Oncol Nurs. 2017:34(3):196-202.

20. Ceppi F, Stephens D, den Hollander BS, Krueger J, Whitlock J, Sung L, et al. Clinical presentation and risk factors of serious infections in children with Down syndrome treated for acute lymphoblastic leukemia. Pediatr Blood Cancer. 2016;63(11):1949-53.

21. Sung L, Aplenc R, Alonzo TA, Gerbing RB, Lehrnbecher T, Gamis AS. Effectiveness of supportive care measures to reduce infections in pediatric AML: a report from the Children's oncology group. Blood. 2013;121(18): $3573-7$.

22. Steliarova-Foucher E, Stiller C, Lacour B, Kaatsch P. International classification of childhood Cancer, third edition. Cancer. 2005;103(7):1457-67.

23. Fisher BT, Zaoutis T, Dvorak CC, Nieder M, Zerr D, Wingard JR, et al. Effect of Caspofungin vs fluconazole prophylaxis on invasive fungal disease among children and Young adults with acute myeloid leukemia: a randomized clinical trial. JAMA. 2019;322(17):1673-81.

24. Alexander S, Fisher BT, Gaur AH, Dvorak CC, Villa Luna D, Dang H, et al. Effect of levofloxacin prophylaxis on bacteremia in children with acute leukemia or undergoing hematopoietic stem cell transplantation: a randomized clinical trial. JAMA. 2018;320(10):995-1004.

25. Kuhn M, Wing J, Weston S, Williams A, Keefer C, Engelhardt A, et al. Classification and Regression Training. 223. https://cran.r-project.org/web/ packages/caret/index.html. Accessed 31 Jan 2019.

26. van Buuren S, Groothuis-Oudshoorn K, Robitzsch A, Vink G, Doove L. Jolani S, et al. Multivariate Imputation by Chained Equations. 2019;174. https:// cran.r-project.org/web/packages/mice/mice.pdf. Accessed 31 Jan 2019.

27. Shah NH, Milstein A, Bagley PhD SC. Making Machine Learning Models Clinically Useful. JAMA. 2019. https://doi.org/10.1001/jama.2019.10306.

28. Morse KE, Bagely SC, Shah NH. Estimate the hidden deployment cost of predictive models to improve patient care. Nat Med. 2020;26(1):18-9.

\section{Publisher's Note}

Springer Nature remains neutral with regard to jurisdictional claims in published maps and institutional affiliations.

Ready to submit your research? Choose BMC and benefit from:

- fast, convenient online submission

- thorough peer review by experienced researchers in your field

- rapid publication on acceptance

- support for research data, including large and complex data types

- gold Open Access which fosters wider collaboration and increased citations

- maximum visibility for your research: over $100 \mathrm{M}$ website views per year

At $\mathrm{BMC}$, research is always in progress.

Learn more biomedcentral.com/submissions 